\title{
Zeitschrift für Psychodrama und Soziometrie
}

\section{Vorwort}

\section{Ulrike Fangauf $\bullet$ Christian Pajek}

Psychodrama ist sinnlich. Wir arbeiten szenisch, mit räumlicher Verdeutlichung interpersonellen und intrapsychischen Geschehens, wir fragen nach optischen Eindrücken, Geräuschen und Gerüchen aus der Erinnerung und setzen im psychodramatischen Spiel den Körper ein, indem wir eine Haltung einnehmen oder Bewegungen ausführen lassen. Dass Psychodrama per se von einer untrennbaren Verknüpfung seelischer und körperlicher Vorgänge ausgeht, zeigt sich immer wieder in der praktischen psychodramatischen Arbeit. Wir erfahren als PsychodramatikerInnen, dass Handeln heilender als Reden ist, dass eine körperliche Darstellung vielfältigere Informationen und wahrhaftigere Emotionen hervorbringt als das bloße Sprechen über eine Situation, dass die Übernahme einer Rolle eine verblüffende psychophysische Wahrnehmung auslösen kann. Doch auch in imaginierten Szenen - etwa in der Telefonseelsorge - gelingt Psychodrama, auch beim Betrachten einer psychodramatischen Inszenierung kann eine (Zuschauer-)katharsis auftreten. Ist der Körper, ist die körperliche Aktion für den Erfolg der psychodramatischen Wirkung nun unerlässlich oder doch nicht? Wie sind körperliches und emotionales Geschehen mit einander verknüpft?

Über die Vielfalt der Beiträge aus unterschiedlichen Feldern stellen wir im vorliegenden Heft einen Teil des breiten psychodramatischen „Antwort-Spektrums“ im Hinblick auf diese Fragestellungen vor.

Gleich zu Beginn lädt uns Sabine Spitzer ein zu einer „Begegnung im virtuellen Raum" und zeigt, wie es gelingen kann, im Internet-Beratungssetting szenisch zu arbeiten ohne reale körperliche Anwesenheit des Gegenübers. Welche Psychodrama-Techniken am PC zum Einsatz kommen, welche Vorteile und Möglichkeiten dieses Mediums besitzt und welche Grenzen und Gefahren zu beachten sind leuchtet der Artikel auf sehr informative und geradezu kurzweilige Art und Weise aus - und ganz nebenbei lernen wir viele feine neue Details über das world wide web.

Ein großer Teil der aktuellen Psychotherapieforschung widmet sich der Fragestellung, welche Zusammenhänge und Wechselwirkungen zwischen der Psyche und dem Körper existieren. So klar auch ist, dass beide Bereiche sich gegenseitig beeinflussen, so divergieren doch die bisherigen Ansätze recht deutlich bezüglich der Frage, inwieweit diese Wechselwirkungen exakt und erschöpfend beschrieben werden können. Unabhängig davon, ob sich diese Fragen in der nächsten Zukunft eindeutig klären lassen, ist festzustellen, dass gerade das Psychodrama sowohl in theoretischer als auch praktischer Sicht eine besondere Bedeutung vor diesem Themenhintergrund einnimmt. Dies ver- 
deutlicht Joachim Becker, dem es gelingt, aus einem sehr großen und unübersichtlichen Feld die relevanten und aktuellen neurobiologischen Erkenntnisse und deren Auswirkungen auf die Psychotherapie verständlich zu beschreiben und darauf aufbauend spezifische Aspekte und Auswirkungen des Psychodramas in neurobiologischer Hinsicht aufzuzeigen.

Von der psychosomatischen Seite her beleuchtet Manfred Stelzig dieses Thema. Zu Beginn seines Beitrags geht er auf die Störung des Verhältnisses zwischen Körper und Seele anhand allgemeiner Überlegungen zur Psychosomatik ein, einschließlich einer diagnostischen Übersicht, und zeigt daran anschließend spezifische Ansätze und Möglichkeiten des tiefenpsychologischen und ressourcenorientierten Psychodramas in der Behandlung psychosomatischer Erkrankungen. Anhand zweier eindrücklicher Falldarstellungen wird deutlich, wie es für PatientIn und TherapeutIn möglich wird, das Wesen der die Symptomatik verursachenden destruktiven Szenen zu erfassen und durch psychodramatische Bearbeitung zu mildern.

Es folgt ein Artikel aus dem Journal of Group Psychotherapy, Psychodrama and Sociometry, den wir, weil er uns so gut gefiel, schon in englischer Sprache vorab unter www.zps-digital.de zur Verfügung gestellt haben. Linda Ciotola beschreibt kurz, aber sehr anschaulich die spezifische psychodramatische Technik des „,Dialogs mit dem Körper“. Obwohl man sich dieses Zwiegespräch sehr gut auch in anderen Settings vorstellen kann, war der Ausgangspunkt für die Entwicklung ihrer Methode die therapeutische Arbeit mit PatientInnen mit Ess- bzw. Traumastörungen, deren Bezug zum Körper massiv gestört war. Linda Ciotolla suchte und fand eine Möglichkeit, über den Rollentausch die Akzeptanz des leidenden Körpers zu erreichen, die dann wieder zu einer positiven Verbindung zwischen Körper und Selbst führt.

Wie vielseitig das Psychodrama im Unterricht angewendet werden kann zeigt uns Bernard Dufeu. Er beschreibt in seinem Beitrag sein spezifisches Verfahren der Psychodramaturgie Linguistique (PDL), wo er durch den modifizierten Einsatz psychodramatischer Techniken ein verbessertes Verständnis der TeilnehmerInnen hinsichtlich der nonverbalen, körperlichen Kommunikationsebene erreicht, was sowohl den Unterricht enorm belebt, als auch die Effektivität des Lernens steigert, ganz zu schweigen vom Zuwachs an Lebensfreude gerade bei spielerischem Lernen mit allen Sinnen.

So, dass wir als Leser in die im wahrsten Sinn des Wortes ,packenden“ Abläufe der Psychodrama-Therapie mit Kindern ganz hineingezogen werden, nimmt uns Alfons Aichinger mit auf den Weg zurück zu körpernahen Sinnen, zum Spiel, in dem auch der Körper der TherapeutInnen ganz besonders gefordert wird. Zunächst nimmt der Psychodrama-Leiter die Signale des kindlichen Körpers auf und übersetzt sie in ein Bild, erlaubt sie und reagiert auf sie. Über neue, adäquate, sinnliche Erfahrungen lernen die Kinder ihren Körper als zentrales emotionales Ausdrucksmittel dadurch neu kennen, schätzen und schützen. So werden Kreativität und Selbstheilungsprozesse in Gang gesetzt, die der physiologischen Entwicklung der Kinder bestens entsprechen.

Der Körper der Therapeuten spielt die Hauptrolle im Beitrag von Klaus Ottomeyer, der in einer kleinen empirischen Studie die Körpererfahrungen von neun PsychodramaTherapeutInnen untersucht. Er verdeutlicht in seinem Beitrag die komplexe gegenseitige Beziehung zwischen TherapeutIn und PatientIn, die Perspektivverschränkung, das Tele und setzt es in Beziehung zu Daniel Sterns aktuellem Konzept des „Gegenwartsmo- 
ments“. Die körperlichen Erfahrungen und intersubjektiven Verschränkungen, die PsychotherapeutInnen mit ihren PatientInnen erleben, bringen oft interessante Wendungen, können aber auch erheblich belasten, umso wichtiger, auch das wird in dem Beitrag deutlich, ist das Sich-Bewusstmachen der in der Therapie möglicherweise auftretenden Körpersensationen.

Eine in der Presse immer wieder gestellte, unbequeme Frage zum Thema Körper ist die, warum so wenige Menschen einen Organspendeausweis unterschreiben. In der Nähe des Themas Tod stoßen wir auf vielfältige intra-psychische, inter-personale und systemisch-gesellschaftliche Rollenkonflikte. Eckhard Frick berichtet in seinem Beitrag über die Möglichkeiten des Psychodramas in Bezug auf den Umgang mit potentiellen Organspendern und Organempfängern. Der Autor beschreibt Möglichkeiten der Anwendungen des psychodramatischen Rollenspiels im klinischen Rahmen sowohl im Hinblick auf die Beantwortung der Frage einer möglichen Organspende als auch im Unterricht mit Studierenden.

Birgit Anna Konteh stellt die Frage, was das Spezifische in der Begleitung von an Krebs erkrankten Menschen ist und worin das besonders Wirksame des Psychodramas liegt. Anhand von Fallbeispielen und theoretischen Überlegungen aus dem psychoonkologischen Bereich erfahren wir mögliche psychische Reaktionen während einer Krebserkrankung und konkrete hilfreiche Interventionen. Dabei ist der Autorin wichtig, keine falschen Erwartungen auf Heilung zu schüren, aber auch nicht einer Lähmung anheim zu fallen. Da Krebs eine Erkrankung ist, die den Menschen in seiner somatischen, psychischen, sozialen und spirituellen Dimension erfasst, ist es auch wichtig, ihn auf allen Ebenen zu begleiten. Das Psychodrama, so verdeutlicht uns die Autorin, kann dazu einen wesentlichen Beitrag leisten, da es über ein Menschenbild, eine Ethik und ein einzigartiges Methodenrepertoire verfügt, das der Lebenskomplexität eines an Krebs erkrankten Menschen gerecht werden kann.

Kristin Scharfetter bringt uns den Körper eines Pferdes näher und die Körpererfahrung des Menschen, der mit dem Pferd in Beziehung tritt. Sie beschreibt sehr einfühlsam, wie ein Pferd als Beziehungspartner und körperliches Medium eine besondere Rollenerfahrung und Reifung für Kinder und Jugendliche entsprechend des psychodramatischen Rollenverständnisses ermöglicht. In zwei Fallvignetten erfahren wir, wie durch den Einsatz eines Pferdes, das gepflegt werden muss, das aber auch stark ist und trägt und das einen eigenen Charakter hat, gerade bei jungen Menschen mit Störungen im sozialen Miteinander und im Selbstwertgefühl, Telefähigkeit und Vertrauen, Einfühlungsvermögen in sich und ein anderes Wesen wachsen und neue glückliche Selbst- und Beziehungerfahrungen gemacht werden können.

In unserer Rubrik „der andere Artikel“ veröffentlicht Christian Pajek die Ergebnisse einer spannenden Untersuchung, in der 19 geschlossene Psychodrama-Gruppen im Rahmen stationärer Psychotherapie hinsichtlich der Entwicklung des Gruppenprozesses bzw. des „Gruppenklimas“ und möglicher Zusammenhänge auf die subjektive Behandlungseinschätzung untersucht wurden. Die Ergebnisse sind erstaunlich und werden schließlich vor dem Hintergrund psychodramatischer und allgemeiner gruppenpsychotherapeutischer Ansätze zum Gruppenprozess diskutiert.

Wir wünschen viel Freude beim Lesen dieser vielfältigen, anregenden Artikel. 